\title{
Encapsulation of proteins and peptides into biodegradable poly(D,L-lactide-co-glycolide) microspheres prolongs and enhances antigen presentation by human dendritic cells
}

\author{
Ying Waeckerle-Men ${ }^{\text {a }}$, Edith Uetz-von Allmen ${ }^{\mathrm{a}, \mathrm{b}}$, Bruno Gander ${ }^{\mathrm{c}}$, Elke Scandella ${ }^{\mathrm{a}}$, \\ Eva Schlosser $^{\mathrm{d}}$, Gunter Schmidtke ${ }^{\mathrm{d}}$, Hans P. Merkle ${ }^{\mathrm{c}}$, Marcus Groettrup ${ }^{\mathrm{a}, \mathrm{b}, \mathrm{d}, *}$ \\ ${ }^{a}$ Research Department, Cantonal Hospital St. Gallen, CH-9007 St. Gallen, Switzerland \\ b Biotechnology Institute Thurgau (BITg), CH-8274 Tägerwilen, Switzerland \\ ${ }^{\mathrm{c}}$ Institute of Pharmaceutical Sciences, Swiss Federal Institute of Technology (ETH) Zürich, CH-8093 Zürich, Switzerland \\ ${ }^{\mathrm{d}}$ Division of Immunology, Department of Biology, University of Constance, \\ PI 101 Universitätsstrasse 10, D-78457 Konstanz, Germany
}

\begin{abstract}
Dendritic cell (DC)-based immunotherapy has been hampered by the lack of suitable methods for antigen delivery. Here, we use biodegradable poly(D,L-lactide-co-glycolide) microspheres (PLGA-MS) as carriers of peptides and proteins for antigen delivery to human monocyte-derived DC (MoDC). Compared to soluble proteins, MHC classes I and II-restricted presentation of PLGA-MS-encapsulated proteins and peptides by MoDC was markedly prolonged and proteins were presented 50-fold more efficiently on class I molecules. The vaccination of mice with DC loaded with PLGA-MS-encapsulated proteins raised strong and persisting cytotoxic T cell responses. In conclusion, antigen delivery via PLGA-MS markedly enhanced the duration of antigen presentation by human MoDC and the potency of DC-based vaccination.
\end{abstract}

Keywords: PLGA microspheres; Dendritic cells; Antigen delivery; Antigen presentation

\section{Introduction}

Dendritic cells (DC) are antigen presenting cells that function as master switches in the regulation of $\mathrm{T}$ cell mediated immune responses [1]. The combination of several properties make them unique which implies that other cell types can not substitute for their function in the priming of $\mathrm{T}$ cells in vivo [2]. One of these properties is a great capacity for the uptake

Abbreviations: Flu, influenza virus; MoDC, monocyte-derived dendritic cells; MS, microspheres; PLGA, poly(D,L-lactide-co-glycolide); TT, tetanus toxoid

* Corresponding author. Tel.: +49 7531 882130; fax: +49 7531883102

E-mail address: Marcus.Groettrup@uni-konstanz.de (M. Groettrup). of soluble and particulate material [3]. A second property is the ability to sense pathogens via toll-like receptors. The contact with prototypic pathogen-derived molecules triggers a third property, i.e. the ability to differentiate from an immature to a mature state. Maturation changes their migratory behaviour such that they migrate from a peripheral site of infection into the $\mathrm{T}$ cell zones of the draining lymph nodes. A further special trait of DC is their ability to process endocytotically acquired material in a way that peptides become cross-presented on MHC class I molecules. The intracellular pathways of cross-presentation in DC are still a matter of investigation [4], but it is remarkable that microparticulate materials are cross-presented by DC much more efficiently than soluble antigens [5]. 
Due to these features and their potency in T cell stimulation, DC have been investigated as cellular vaccines in immunotherapy against tumors and infectious diseases. Inoculation of antigen-loaded DC is one of the most powerful ways to trigger a $\mathrm{T}$ cell response, and numerous phase I/II clinical trials have been published that were based on vaccination with antigen-loaded autologous DC [6]. Protocols to generate large numbers of immature DC from peripheral blood monocytes by differentiation in IL-4 and GM-CSF [7] as well as procedures to reliably mature and cryopreserve monocyte derived DC (MoDC) [8] have greatly facilitated this approach. While it emerged that DC-based vaccination is feasible and well tolerated, it became evident that the CTL responses in patients were not overwhelming, and clinical responses were confined to a small subset of patients.

One likely reason, why DC-based tumor immunotherapy in humans has remained inefficient, is a lack of suitable methods for antigen delivery to MoDC. Most trials published to date relied on externally pulsing MoDC with synthetic class I peptides. Because of the rapid turn over of class Ipeptide complexes on the cell surface, the duration of $\mathrm{T}$ cell stimulation in vivo is probably not long enough. In mice, it has been shown that the ability of peptide-pulsed DC to stimulate antigen-specific CTL was lost within a day after injection, whereas DC that endogenously expressed the same antigen could activate antigen specific CTLs for more than 2 days [9]. The external loading with class I epitopes is also unsatisfactory, because it confines immunotherapy to patients expressing a certain restriction element and because antigenspecific help is not provided. It would be optimal to charge MoDC with entire protein antigens, that are likely to contain several MHC classes I and II-restricted epitopes, and deliver the proteins in a form such that they can be taken up efficiently by DC and provide T cell epitopes over an extended time period.

In order to achieve these aims, we have investigated biodegradable poly(D,L-lactide-co-glycolide) microspheres (PLGA-MS) as carriers of peptides and proteins for antigen delivery to human MoDC. PLGA-MS are microparticles that are slowly hydrolyzed in an aqueous environment and have been used as drug delivery vehicles for sustained release in humans [10]. PLGA-MS have previously been investigated as single dose vaccine formulations which elicited potent antibody [11-13] as well as Th and CTL responses [14-17] against encapsulated peptides or proteins. The PLGA polymer used in this study is composed of $50 \%$ glycolate and $50 \%$ lactate and is hydrolyzed in aqueous media over a period of approximately 20-30 days during which encapsulated peptides and proteins are released [18]. By spray-drying, peptides, proteins or DNA can be microencapsulated into PLGA-MS of a size range of $0.5-5 \mu \mathrm{m}$. Particles of such sizes can be efficiently phagocytosed by murine macrophages and DC in vitro and in vivo $[19,20]$, and antigen presentation of T cell epitopes on MHC classes I and II molecules has been demonstrated [5,21,22].
Previously, we have shown that MoDC, which were generated in serum-free medium according to a protocol suited for clinical application [23], readily take up PLGAMS without adverse effects on their phenotype, cytokine production, allostimulatory potential, survival, and migration [20]. In this study, we investigated MHC class Irestricted cross-presentation and MHC class II-restricted direct presentation of peptides and proteins that were taken up by human MoDC in a PLGA-MS encapsulated form. We show that this technique of antigen delivery allows efficient and long-lasting antigen presentation by MoDC in vitro and the generation of strong CTL responses in vivo.

\section{Materials and methods}

\subsection{Materials and cell culture reagents}

PLGA 50:50 of approx. 14 kDa and carrying uncapped hydroxyl and carboxyl end-groups (Resomer ${ }^{\circledR}$ RG502H) was purchased from Boehringer Ingelheim (Ingelheim, Germany), AIM-V medium from Invitrogen (Groningen, The Netherlands), MDM medium and FCS from Sigma (Buchs, Switzerland) and Ficoll-Paque ${ }^{\circledR}$ from Pharmacia (Uppsala, Sweden). Human GM-CSF (Leukine ${ }^{\circledR}$ ) was obtained from Immunex (Seattle, WA, USA), Human recombinant cytokines IL-4, TNF- $\alpha$, IL-1 $\beta$, and IL-6 were bought from CellGenix (Freiburg, Germany). Murine GM-CSF was kindly provided by Novartis (Vienna, Austria). LPS (from Salmonella abortus equi) and $\beta$-galactosidase ( $\beta$-Gal) were bought from Sigma. PGE2 was obtained from Pharmacia \& Upjohn (Dübendorf, Switzerland) and Tetanus toxoid from Berna Biotech (Berne, Switzerland). HLA-A*0201-restricted peptide FluM ${ }_{58-66}$ (Influenza A M1 protein 58-66, amino acid sequence GILGFVFTL), HLA-DR5-restricted peptide tt30 (tetanus toxrn947-967, FNNFTVSFWLRVPKVSASHLE) and $\mathrm{H}-2 \mathrm{~K}^{\mathrm{b}}$-restricted peptide I8V ( $\beta$-Gal 497-504, ICPMYARV) were synthesized by Echaz Microcollections (Reutlingen, Germany). PE-labeled I8V/H-2 $\mathrm{K}^{\mathrm{b}}$ tetramer was a gift from Philippe Krebs (Institute of Experimental Immunology, University Hospital Zurich, Switzerland).

\subsection{Preparation of GST-FluM fusion protein}

A glutathion- $S$-transferase-influenza virus matrix (GSTFluM) expression construct was kindly provided by Dr. Stephan Ludwig, University of Würzburg. BL21 cells were transfected with the GST-FluM expression plasmid and grown at $28^{\circ} \mathrm{C}$. GST-FluM expression was induced with $0.5 \mathrm{mM}$ isopropyl $\beta$-D-1-thiogalactopyranoside (IPTG) for $6 \mathrm{~h}$ and cells were pelleted and lysed by ultrasound treatment and extracted with 1\% Triton X-100. The insoluble debris was removed by centrifugation and GST-FluM protein was purified over a glutathion-sepharose matrix (Amersham 


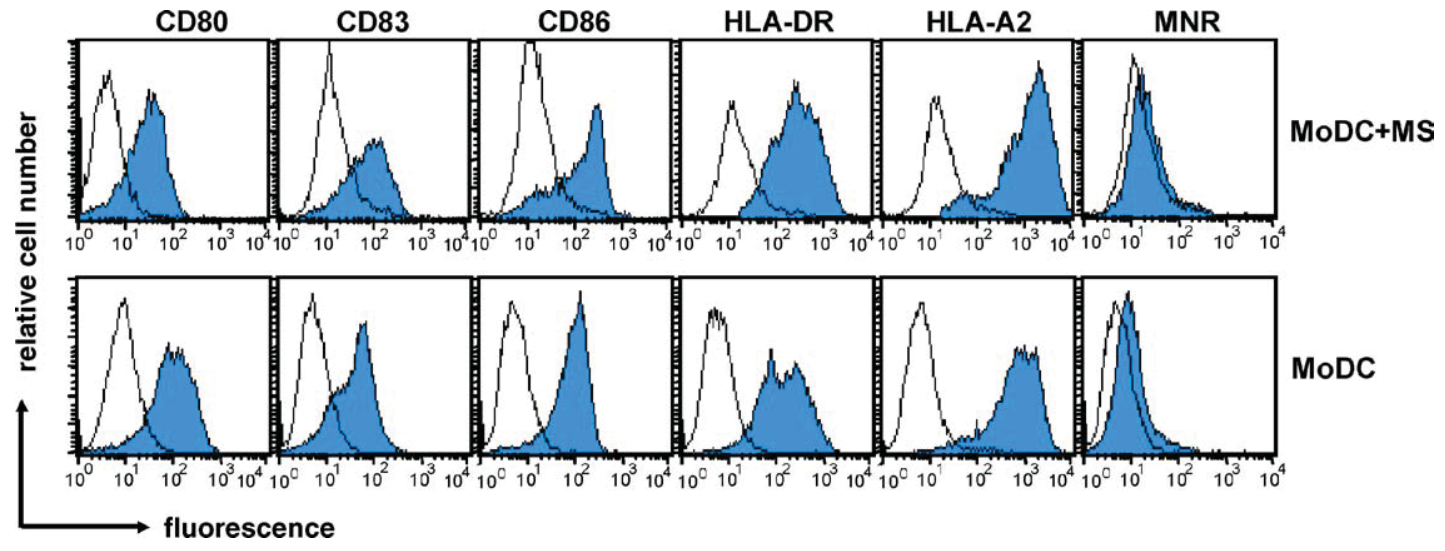

Fig. 1. Impact of PLGA-MS uptake on the cell surface expression of DC differentiation markers and the ability to mature MoDC. Untreated immature human MoDC (MoDC) or MoDC that had taken up PLGA-MS (MoDC + MS) were matured with a cocktail of pro inflammatory cytokines (TNF- $\alpha$, IL-1 $\beta$, IL-6, PGE2) for two days and subsequently analysed for surface expression of CD80, CD83, CD86, HLA-DR, HLA-A2, and the mannose receptor (MNR) by flow cytometry (filled gray lines). Open black histograms represent staining with isotype matched control mAbs. Data represent one out of six independent experiments which all gave similar results.

Biosiences, Freiburg, Germany) according to standard protocols.

\subsection{Microencapsulation of antigens}

All antigens used in this study were microencapsulated into PLGA 50:50 of a size of $0.5-5 \mu \mathrm{m}$ by spray-drying as previously described [20]. Briefly, 10 or $20 \mathrm{mg}$ of antigen were dissolved in $1 \mathrm{ml}$ of phosphate buffered saline and $1 \mathrm{~g}$ of PLGA in $20 \mathrm{ml}$ ethyl formate. The two solutions were mixed and homogenized under ultrasonication $(20 \mathrm{kHz}$, 50 W, VibraCell VC50T, Sonics \& Materials, Danbury, CT) during $2 \times 30 \mathrm{~s}$ under cooling on ice. This w/o-dispersion was spray-dried (Mini Spray-Dryer 191, Büchi, CH-Flawil) at a flow rate of $2 \mathrm{ml} / \mathrm{min}$, and at inlet and outlet temperatures of 48 and $40^{\circ} \mathrm{C}$, respectively. The spray-dried MS were washed out of the spray-dryer's cyclone and product recipient with $0.1 \%$ poloxamer 188 solution, collected on a cellulose acetate membrane filter, washed with water to remove non-encapsulated protein, and dried under reduced pressure (20 mbar) for $24 \mathrm{~h}$. The antigen contents of the resulting MS were as follows: MS-tt30, $6.7 \mu \mathrm{g} / \mathrm{mg}$; MS-TT, $12 \mu \mathrm{g} / \mathrm{mg}$; MS-Flu peptide, $5.7 \mu \mathrm{g} / \mathrm{mg}$; MS-FluM, $4.2 \mu \mathrm{g} / \mathrm{mg}$; MS-I8V, $6 \mu \mathrm{g} / \mathrm{mg} ; \mathrm{MS}-\mathrm{PGal}, 4.7 \mu \mathrm{g} / \mathrm{mg}$.

\subsection{Generation of DC and antigen loading with PLGA-MS}

Human MoDC were generated from peripheral blood mononuclear cells (PBMC) under clinical conditions as previously described [20]. After 5 days of differentiation in IL-4 and GM-CSF, cells were collected for flow cytometric analysis as described elsewhere [20]. The cells showed a typical phenotype of immature DC $\left(\mathrm{CD}_{1} \mathrm{a}^{+}, \mathrm{CD}^{-}, \mathrm{CD} 11 \mathrm{c}^{+}\right.$, $\mathrm{CD}_{14}^{-}, \mathrm{CD}^{-} 6^{-}, \mathrm{CD}_{20}^{-}, \mathrm{CD} 40^{+}, \mathrm{CD} 80^{+}, \mathrm{CD}^{-} 3^{-}, \mathrm{CD}^{+} 6^{+}$, $\mathrm{CD}^{+} 5^{+}, \mathrm{MNR}^{+}, \mathrm{MHC}$ classes I and II molecules positive, and $\mathrm{CCR}^{-}$), and were hence designated immature MoDC. Where indicated, a cocktail containing $20 \mathrm{ng} / \mathrm{ml} \mathrm{TNF-} \alpha$, $10 \mathrm{ng} / \mathrm{ml} \mathrm{IL-1 \beta ,} 1000 \mathrm{U} / \mathrm{ml} \mathrm{IL-6,} \mathrm{and} 1 \mu \mathrm{g} / \mathrm{ml}$ PGE2 was added to immature MoDC previously loaded or not with soluble or encapsulated antigens. Upregulation of CD40, CD80, CD83, CD86, MHC class II molecules and CCR7, and downregulation of MNR was observed after 2 days of maturation; the cells were hence called mature MoDC (Fig. 1).

Murine BM-DC were generated as previously described [20]. Where indicated, $1 \mu \mathrm{g} / \mathrm{ml}$ LPS was added to Ag-loaded BM-DC for $24 \mathrm{~h}$ for further DC activation.

To deliver MS-encapsulated antigens to human MoDC or mouse BM-DC, the indicated amounts of PLGA-MS were first dispersed in cell culture medium by ultrasonication for $1 \mathrm{~min}$ in order to disintegrate microparticulate aggregates and obtain a very homogenous suspension of primary microsphere particles. Then these microsphere suspensions were added to immature MoDC or BM-DC cultures. After $4 \mathrm{~h}$ of incubation at $37^{\circ} \mathrm{C}$, about $50-60 \%$ of DC had ingested at least one PLGA particle (data not shown). Where indicated, proinflammatory cytokines (for MoDC) or LPS (for BM-DC) were added to activate DC for another 2 days before analysis of antigen presentation.

\subsection{Generation of $T$ cell lines}

Human $\mathrm{CD}^{+} \mathrm{T}$ cell lines SK.30 (specific to tt30 peptide) and KR.TT (specific to tetanus toxoid protein (TT)) were generated in our laboratory. Briefly, $\mathrm{CD} 4^{+}$cells from a HLA-DR $5^{+}$donor were magnetically isolated by $\mathrm{CD} 4^{+}$ MACS microbeads and resuspended in $\mathrm{T}$ cell medium (IMDM medium supplemented with 10\% AB serum, 1\% HEPES, $100 \mathrm{U} / \mathrm{ml}$ penicillin, and $100 \mu \mathrm{g} / \mathrm{ml}$ streptomycin) at $2 \times 10^{6} / \mathrm{ml}$ and cultured in 24-well plates. These cells were weekly restimulated with irradiated (30 Gy) autologous PBMC pulsed with either $10 \mu \mathrm{g} / \mathrm{ml}$ tt30 or TT. After 4-6 


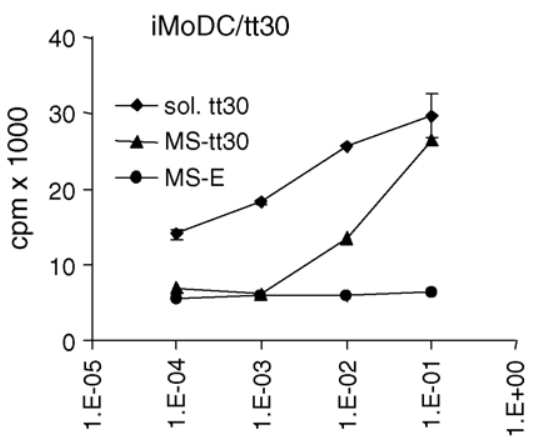

(A)

$\mathrm{tt} 30$ concentration $(\mu \mathrm{g} / \mathrm{ml})$

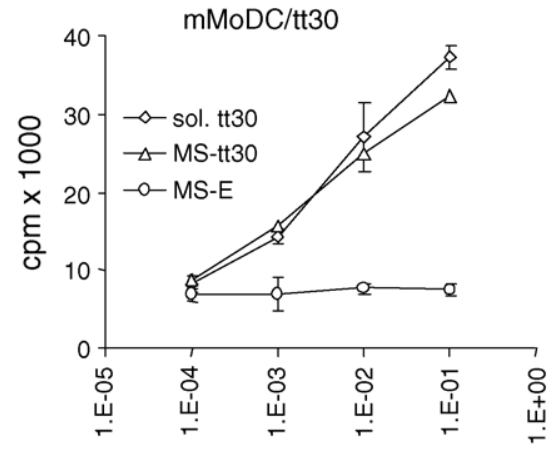

(C)

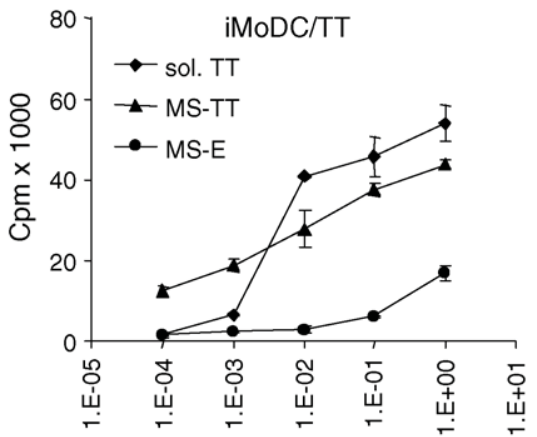

(E)

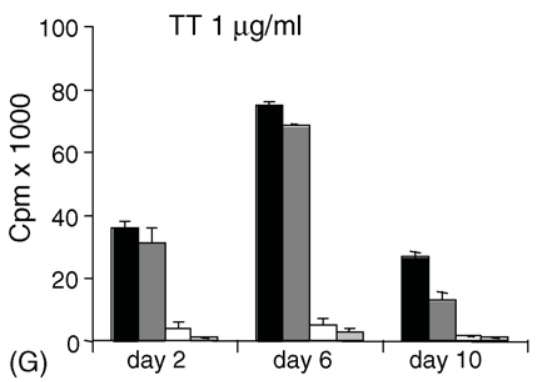

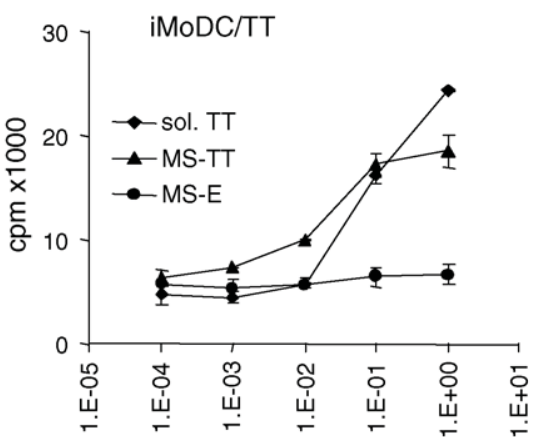

(B)

TT concentration $(\bar{\mu} \mathrm{g} / \mathrm{ml})^{-}$

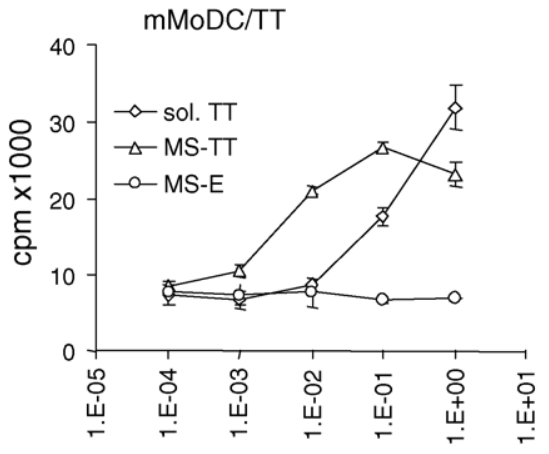

(D) $\quad \mathrm{TT}$ concentration $(\mu \mathrm{g} / \mathrm{ml})$

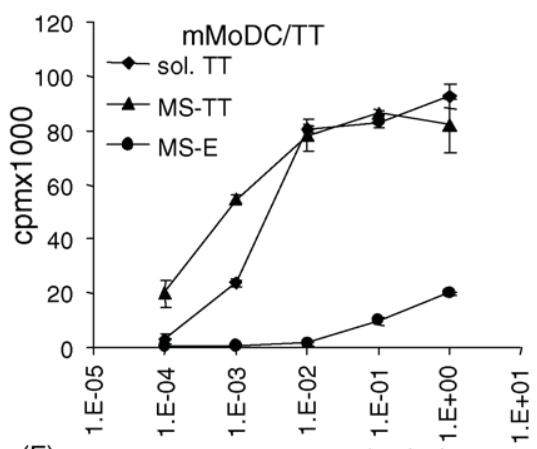

(F)

TT concentration $(\mu \mathrm{g} / \mathrm{ml})^{-}$

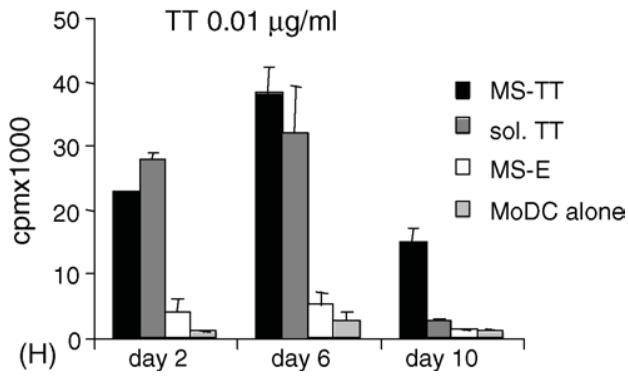

Fig. 2. Presentation of PLGA-encapsulated tetanus toxoid (TT) antigens by human MoDC to specific CD4 ${ }^{+} \mathrm{T}$ cell lines. Immature human MoDC were loaded with soluble (sol) or PLGA-MS-encapsulated (MS-) tt30 peptide or TT protein for 2 days at $37^{\circ} \mathrm{C}$ as indicated. MoDC were either left immature (iMoDC, $\mathrm{A} / \mathrm{B}$ ), or first loaded with the antigens for $4 \mathrm{~h}$ at $37^{\circ} \mathrm{C}$ and then proinflammatory cytokines were added for maturation for 2 days (mMoDC, C/D). MoDC were co-cultured with tt30-specific CD4 ${ }^{+} \mathrm{T}$ cells SK.30 and proliferation was measured by ${ }^{3} \mathrm{H}$-thymidine incorporation (Fig. 2A-D). (E/F) MoDC were loaded with soluble TT or MS-TT with (F) or without maturation (E) as described above. MoDC were then co-cultured with the TT specific CD4 ${ }^{+}$T cell line KR.TT and T cell proliferation was determined. (G/H) MoDC were loaded with a high $(1 \mu \mathrm{g} / \mathrm{ml}, \mathrm{G})$ or low dose $(0.01 \mu \mathrm{g} / \mathrm{ml}, \mathrm{H})$ of TT in soluble or in PLGA-MS-encapsulated form and matured. KR.TT cells were added to TT-loaded MoDC on day 2, 6 or 10, respectively, to evaluate the kinetics of TT presentation by MoDC. All data are shown as the mean cpm from duplicate cultures \pm S.E.M. and summarized from four independent experiments. Empty PLGA-MS (MS-E) or untreated MoDC (MoDC alone) served as negative controls as indicated. 
restimulations, cells were tested for $\mathrm{T}$ cell proliferation with MoDC pulsed with relevant antigens (data not shown).

Human $\mathrm{CD}^{+}$CTL line Flu.2 specific to Flu ${ }_{58-66}$ peptide was generated from PBMC of an HLA-A ${ }^{*} 0201^{+}$donor who had received Flu vaccination recently. In brief, $\mathrm{CD}^{+}$ cells were magnetically isolated from PBMC, suspended in T cell medium at $2 \times 10^{6} / \mathrm{ml}$, and cultured in 24 -well plates. Autologous matured MoDC were pulsed with $5 \mu \mathrm{g} / \mathrm{ml}$ Flu peptide for $2 \mathrm{~h}$ at $37^{\circ} \mathrm{C}$, irradiated (30 Gy) and added to CD8 ${ }^{+}$ cell culture at $5 \times 10^{5} /$ well. Autologous PBMC were irradiated (35 Gy) and used as feeder cells ( $4 \times 10^{6} /$ well). After six restimulations, $\mathrm{CD}^{+} \mathrm{CTL}$ were tested for their antigen specificity with Flu/HLA-A2-tetramer staining and for cytotoxicity with ${ }^{51} \mathrm{Cr}$ release assay. A portion of $>50 \%$ of CTL were Flu/HLA-A2-tetramer positive and demonstrated a high level of Flu-peptide-specific cytotoxicity (data not shown).

\subsection{Determination of antigen presentation by human MoDC in vitro}

To present MHC class II antigens to $\mathrm{CD}^{+} \mathrm{T}$ cells, MoDC were loaded with soluble or PLGA-MS-encapsulated tt30 or TT at various concentrations as indicated in Fig. 2. Subsequently, MoDC were resuspended in $\mathrm{T}$ cell medium and seeded into 96-well flat bottom microplates at $5 \times 10^{4}$ MoDC in $100 \mu$ l per well. SK.30 (Fig. 2A-D) or KR.TT (Fig. 2E-H) T cells were added to the MoDC culture at $1 \times 10^{5}$ per well $(100 \mu \mathrm{l})$ for co-incubation in duplicates at the indicated time points. After 3 days of co-culture, ${ }^{3} \mathrm{H}$-thymidine $(1 \mu \mathrm{Ci} /$ well) was added for $16-18 \mathrm{~h}$, and $\mathrm{T}$ cell proliferation was determined by ${ }^{3} \mathrm{H}$-thymidine incorporation.

To study the presentation of Flu antigens to $\mathrm{CD} 8^{+} \mathrm{CTL}$ by MoDC (Fig. 3), soluble or PLGA-MS-encapsulated Flu peptide or GST-FluM protein were co-incubated with immature MoDC, and subsequently, maturation stimuli were provided as described above. Antigen-loaded matured MoDC were collected, washed and resuspended in $\mathrm{T}$ cell medium and seeded into 96-well U-bottom microplates. Flu.2 CTL were added to the antigen-loaded MoDC culture at the indicated time points. After $24 \mathrm{~h}$ of co-culture, supernatants were collected and analyzed for IFN- $\gamma$ content with a human IFN- $\gamma$ ELISA kit (BD OptEIA ${ }^{\mathrm{TM}}$, BD Biosciences Pharmingen, San Diego, USA).

\subsection{Immunization of mice with mouse BM-DC loaded with soluble or PLGA-MS-encapsulated antigens}

Murine CD11c ${ }^{+}$BM-DC prepared from C57BL/6 mice were loaded with soluble or PLGA-MS-encapsulated peptide I8V ( $\beta$-gal $497-504)$ or $\beta$-gal protein and activated with LPS, as indicated in Fig. 5. After washing to remove free antigens, $3 \times 10^{5}$ Ag-loaded BM-DC were injected into B6 mice intravenously at the tail vein (three mice per group).
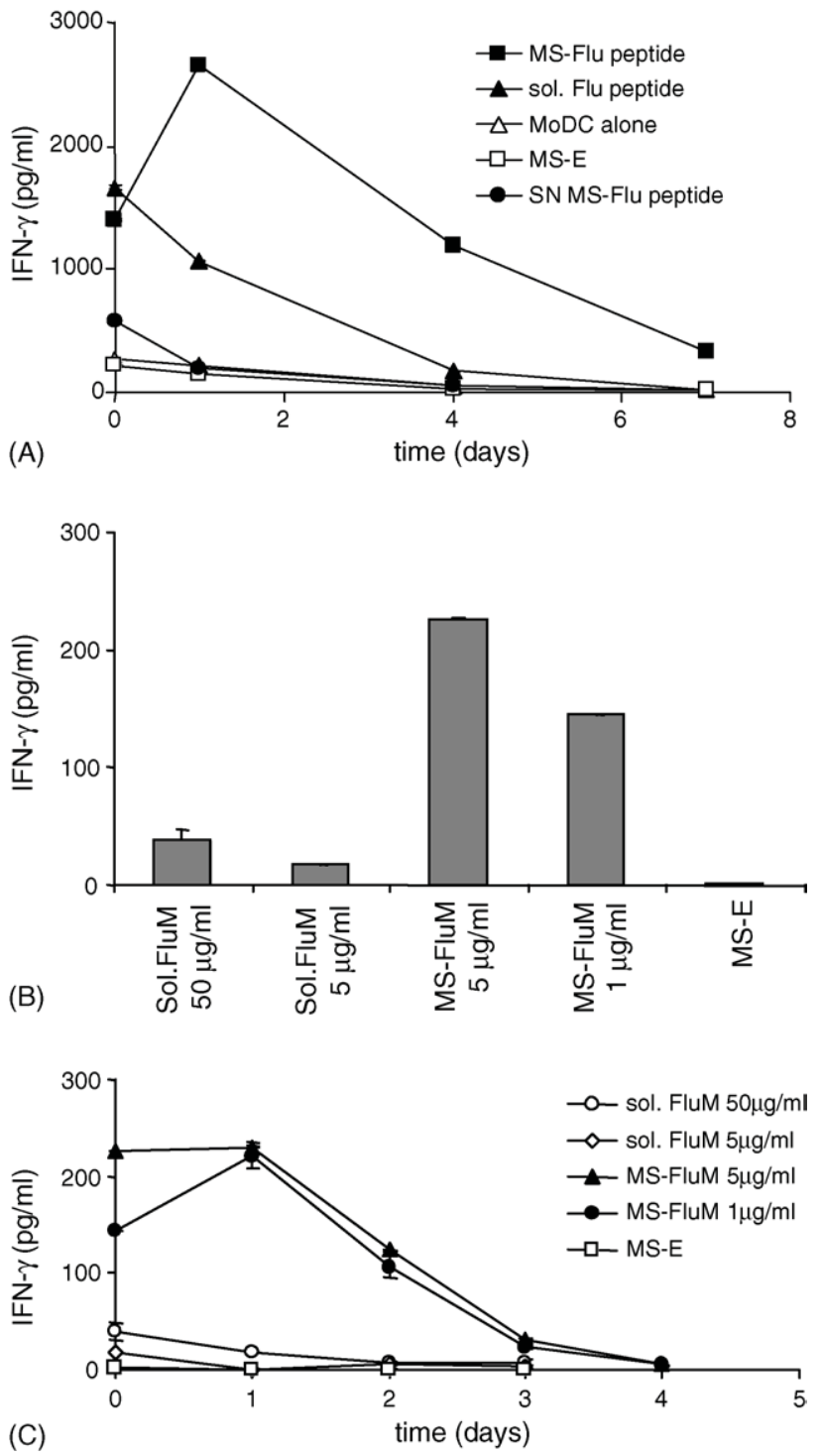

Fig. 3. Encapsulation of influenza matrix peptide and protein in PLGA-MS prolongs presentation of the M1 epitope $\left(\right.$ FluM $_{58-66}$ ) by human MoDC. (A) MoDC were loaded with $1 \mu \mathrm{g} / \mathrm{ml}$ soluble M1 epitope (sol. Flu peptide) or the same amount of PLGA-MS-encapsulated Ml peptide (MS-Flu peptide) for $4 \mathrm{~h}$ at $37^{\circ} \mathrm{C}$ followed by maturation for 2 days. In addition, MS-Flu peptide particles (equivalent to $1 \mu \mathrm{g} / \mathrm{ml}$ of soluble peptide) were kept in cell culture medium for $24 \mathrm{~h}$, and the supernatant was used for incubation with MoDC (SN MS-Flu peptide). The M1 peptide specific CD8 ${ }^{+}$CTL line Flu. 2 was added to MoDC immediately after antigen loading (day 0) or on day 1,4 or 7 thereafter. Supernatants were collected after $24 \mathrm{~h}$ of co-incubation for measurement of IFN- $\gamma$ production by ELISA. MoDC without antigen (MoDC alone) or loaded with empty MS (MS-E) were used as control. (B) Immature MoDC were loaded for $4 \mathrm{~h}$ with different amounts of soluble GST-FluM protein (sol. FluM) or with PLGA-MS containing equivalent or 10-fold higher amounts of soluble GST-FluM (MS-FluM) as indicated, followed by MoDC maturation for 2 days. Flu.2 CTL were added to the FluM-loaded matured MoDC $\left(5 \times 10^{4} \mathrm{CTL}\right.$ to $\left.1 \times 10^{4} \mathrm{MoDC}\right)$ and then supernatants were collected after $24 \mathrm{~h}$ for assessment of IFN- $\gamma$ production. (C) MoDC were prepared as described in B and Flu.2 CTL were added to the MoDC either immediately (day 0 ), or on day $1,2,3$, and $4\left(5 \times 10^{4} \mathrm{CTL}\right.$ to $1 \times 10^{4} \mathrm{MoDC}$ ) as described in B. Supernatants were collected after $24 \mathrm{~h}$ for measurement of IFN- $\gamma$ production. The data are shown as the mean of duplicate cultures \pm S.E.M. averaged from three experiments. 


\subsection{Determination of I8V-specific CTL by $I 8 V / H-2 K^{b}$ tetramer staining}

Three to five drops of blood were collected from immunized mice by tail bleeding at the indicated time points. Blood was collected in FACS-EDTA buffer (PBS containing $2 \%$ FCS, $0.5 \mathrm{M}$ EDTA and $0.03 \% \mathrm{NaN}_{3}$ ), and stained with PE-labeled I8V/H-2K $\mathrm{K}^{\mathrm{b}}$ tetramer for $10 \mathrm{~min}$ at $37^{\circ} \mathrm{C}$, and subsequently with FITC-labeled anti-CD8 mAb for $20 \mathrm{~min}$ at $4{ }^{\circ} \mathrm{C}$. Erythrocytes were then lysed with BD FACS ${ }^{\mathrm{TM}}$ Lysing Solution (BD Biosciences, Erembodegem, Belgium), cells were washed once and resuspended in $500 \mu$ l FACS buffer for flow cytometric analysis with a FACScan ${ }^{\circledR}$ flow cytometer (Becton Dickinson).

\subsection{Cytolytic assay}

${ }^{51} \mathrm{Cr}$ release assay was used to determine the function of $\mathrm{I} 8 \mathrm{~V} / \mathrm{H}-2 \mathrm{~K}^{\mathrm{b}}$ tetramer positive CTL. EL-4 target cells were either pulsed with $1 \mu \mathrm{g} / \mathrm{ml}$ of I8V peptide or left unpulsed and were labeled with ${ }^{51} \mathrm{Cr}$ for $1 \mathrm{~h}$ at $37^{\circ} \mathrm{C}$. After washing, labeled target cells (2000 cells in $100 \mu \mathrm{l}$ ) were added to various numbers of effector cells (splenocytes from immunized mice after one cycle of re stimulation) in Ubottom 96-well microplates. Chromium release was measured in the supernatant $(100 \mu \mathrm{l})$ after $4 \mathrm{~h}$ of incubation at $37^{\circ} \mathrm{C}$

\section{Results}

\subsection{Delivery of tetanus toxoid antigens to human MoDC via PLGA-MS prolongs MHC class II-restricted antigen presentation}

To assess the efficiency of MHC classes I and II-restricted antigen presentation by human MoDC, we first determined the cell surface expression of MHC molecules and DC maturation markers before and after uptake of PLGA-MS. In agreement with previous investigations [20], the surface expression of CD80, CD83, CD86, HLA-DR, HLAA2 and CCR7 was not altered after uptake of PLGAMS by immature MoDC. Moreover, PLGA-MS uptake did not induce MoDC maturation, but MoDC loaded with PLGA-MS could be matured with a cocktail of proinflammatory cytokines (TNF- $\alpha$, IL-1 $\beta$, IL-6, PGE2), as indicated by the upregulation of aforementioned markers (Fig. 1).

To assess class II-restricted presentation, immature MoDC were loaded with different concentrations of the HLA-DR5restricted tt30 peptide from tetanus toxoid protein (TT) either in soluble or PLGA-MS-encapsulated form. Antigenpulsed MoDC were then left immature or were matured with the proinflammatory cytokines (Fig. 2). MoDC were then incubated with the HLA-DR5/tt30 specific CD4 ${ }^{+} \mathrm{T}$ helper cell line SK30 and T cell proliferation was monitored (Fig. 2A-D). For immature MoDC, loading with soluble tt30 peptide seemed to be more efficient than with PLGA-MSencapsulated tt30 (MS-tt30, Fig. 2A) but this difference was not observed with matured MoDC where both methods were equally efficient (Fig. 2C). When TT protein was used as antigen, 10-fold lower amounts of TT were required for tt30 presentation when it was delivered via PLGA-MS (MS-TT, Fig. 2D). To substantiate this result, we also used the oligoclonal TT specific $\mathrm{CD}^{+}$cell line KR.TT to monitor TT presentation (Fig. 2E-H). Proliferation of KR.TT cells could be detected when MoDC were loaded with $0.1 \mathrm{ng} / \mathrm{ml}$ PLGAMS-encapsulated TT, while at least 10-fold larger quantities (1.0-10 ng) were needed to achieve an equivalent stimulation with soluble TT (Fig. 2E and F).

Next, we performed a kinetic analysis of TT presentation. Immature MoDC were loaded with either soluble or PLGA-MS-encapsulated TT at a saturating concentration of $1.0 \mu \mathrm{g} / \mathrm{ml}$ (Fig. 2G) or at a limiting concentration of $0.01 \mu \mathrm{g} / \mathrm{ml}$ (Fig. $2 \mathrm{H}$ ). MoDC were then matured with proinflammatory cytokines, and TT-presentation was recorded with KR.TT cells after several days. Antigen delivery of TT via PLGA-MS was clearly superior to pulsing with soluble protein, which became apparent on day 10 after antigen loading with $0.01 \mu \mathrm{g} / \mathrm{ml}$ TT when the presentation of PLGA-MSencapsulated TT persisted, whereas presentation of soluble TT had waned.

\subsection{Prolongation and enhancement of MHC class I-restricted cross-presentation by encapsulation of influenza virus matrix protein and peptide}

We investigated cross-presentation of PLGA-MSencapsulated proteins and peptides using the influenza virus matrix protein (FluM) and the HLA-A*0201 restricted M1 epitope (FluM58-66) as model antigens. First, external loading of mature MoDC with the M1 epitope was compared to loading immature MoDC with PLGA-MS-encapsulated M1 (MS-Flu peptide) followed by DC maturation in vitro. M1-presentation was monitored by IFN- $\gamma$ production of an M1/HLA-A*0201-specific CTL line during several days after antigen pulsing (Fig. 3A). Interestingly, MS-Flu peptide presentation persisted up to day 7 while presentation ceased on day 4 after external peptide loading. The presentation of externally loaded peptide declined continuously whereas presentation of PLGA-MS-encapsulated FluM peptide was maximal one day after delivery which coincides with the so called burst release of encapsulated material from PLGA-MS known to occur within the first day of incubation. To ascertain that M1 presentation from PLGA-MS was not due to peptide release and external binding to class I molecules during coincubation, we incubated MoDC with the supernatant of MSFlu peptide which was obtained after $24 \mathrm{~h}$ of release in $\mathrm{T}$ cell medium with the same concentration as used for MS-loading. Since only negligible antigen presentation was detected (Fig. 3A, SN MS-Flu peptide), it appears that Ml-loading of HLA-A*0201 molecules of MoDC occurred within the cells. 

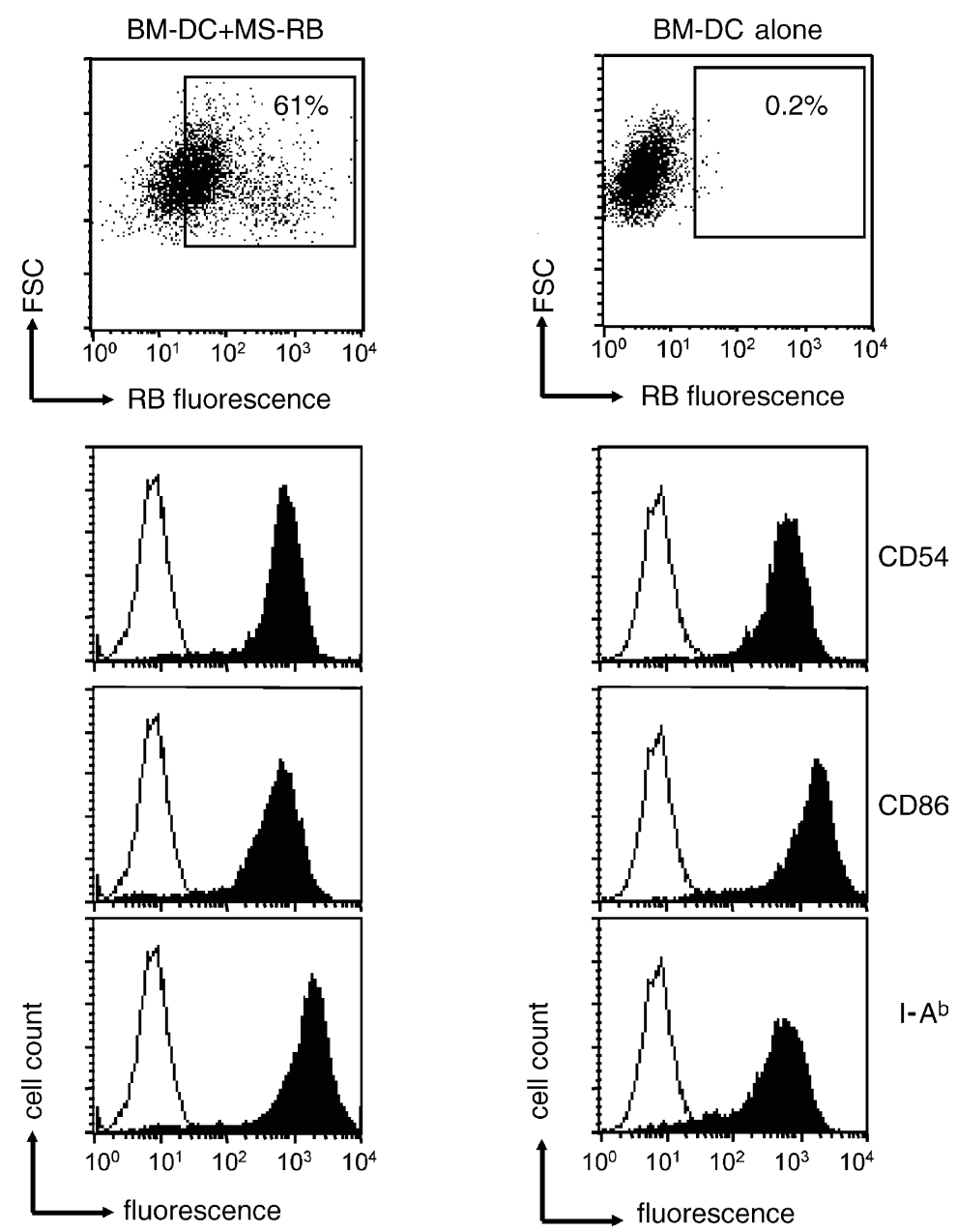

Fig. 4. The uptake of PLGA-MS by murine BM-DC. CD11 ${ }^{+}$murine BM-DC were loaded with PLGA-MS that contained the fluorescent dye Rhodamine B (MS-RB) and were then activated overnight with LPS followed by the analysis of MS-RB uptake by flow cytometry (upper panels). Numbers within the dot plot gates represent percentage of BM-DC that were positive for RB fluorescence. The phenotype of fluorescent CD11 $\mathrm{c}^{+} \mathrm{BM}$-DC was determined by surface staining with mAbs specific for CD54, CD86, and H2-I-A ${ }^{\mathrm{b}}$ followed by flow cytometric analysis (filled black lines). Open black lines represent BM-DC without Ab staining. BM-DC without MS loading were used as negative control. Data represent one out of three independent experiments which all gave similar results.

To examine how a model protein can be cross-presented from PLGA-MS, a recombinant GST-influenza matrix fusion protein (FluM) was encapsulated into PLGA-MS (MS-FluM) and immature MoDC were incubated with MSFluM at different concentrations and subsequently matured. For comparison, untreated MoDC were incubated with the equivalent amount of soluble FluM and presentation of the M1 epitope was monitored (Fig. 3B). Remarkably, $50 \mu \mathrm{g} / \mathrm{ml}$ of soluble FluM was not sufficient to achieve a level of CTL stimulation obtained with only $1 \mu \mathrm{g} / \mathrm{ml}$ of FluM after uptake via PLGA-MS. A kinetic experiment was then performed to compare stimulation of M1-specific CTL at different time points after MoDC had been loaded with either soluble FluM or MS-FluM. As shown in Fig. 3C, presentation of soluble FluM protein was very low and vanished completely after one or two days, whereas presentation of MS-FluM lasted for at least 3 days.

\subsection{DC-based vaccination of mice elicits strong CTL responses when a protein or peptide is delivered to $D C$ via PLGA-MS}

To investigate the antigen specific CTL response elicited by PLGA-MS treated DC in vivo we switched to the mouse model. First, we tested whether murine bone marrow-derived DC (BM-DC) phagocytose PLGA-MS in vitro as efficiently as human MoDC. BM-DC were incubated overnight with PLGA-MS that contained the fluorescent dye Rhodamine B (MS-RB), and the portion of fluorescent cells was determined by flow cytometry (Fig. 4, top panels). About $60 \%$ of the BM-DC were fluorescent after overnight incubation which is even a bit higher than what we have recently observed in similar experiments for human MoDC where about $45 \%$ of the cells had engulfed PLGA-MS [20]. To monitor the effect of PLGA-MS uptake on the cell surface phenotype of BM-DC 
(A)
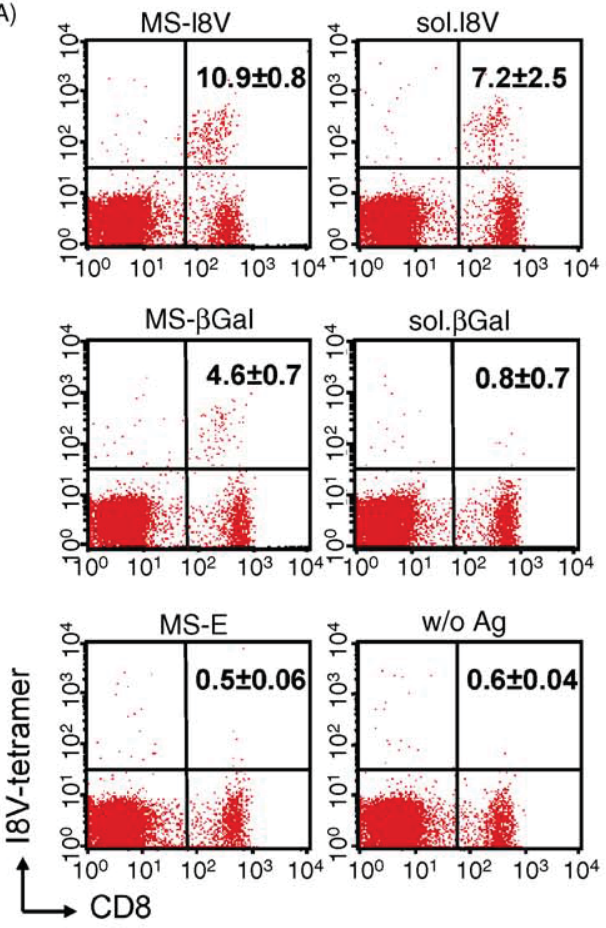

(C)

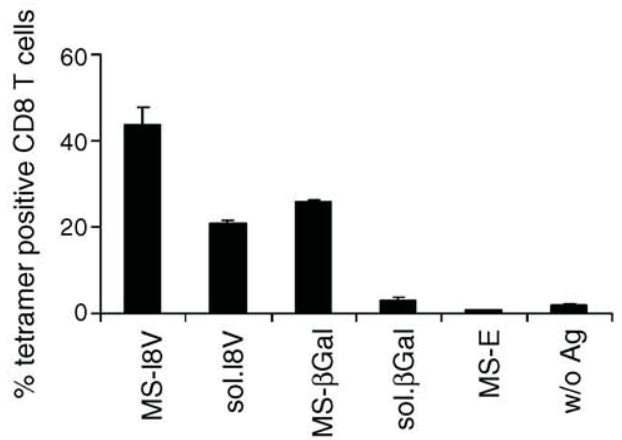

(B)

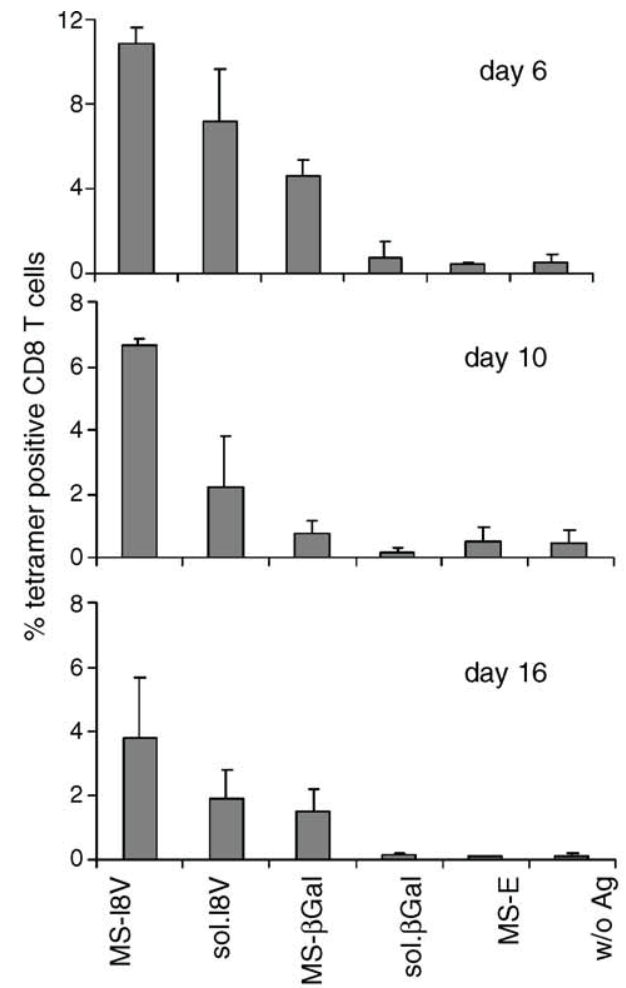

(D)
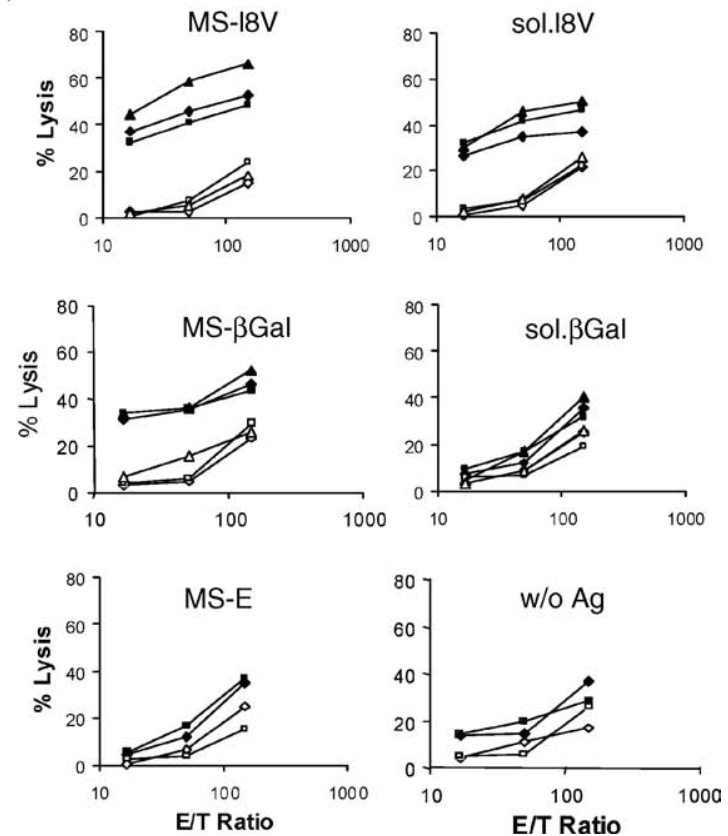

Fig. 5. Antigen loading of BM-DC with PLGA-MS leads to enhanced CTL responses in vivo. (A and B) Murine CD11 ${ }^{+}$BM-DC prepared from C57BL/6 mice were loaded with $1 \mu \mathrm{g} / \mathrm{ml} \beta \mathrm{Gal}_{497-504}$ peptide (I8V) encapsulated in PLGA-MS (MS-I8V) or in soluble form (sol.I8V). Alternatively, BM-DC were loaded with $1 \mu \mathrm{g} / \mathrm{ml}$ of PLGA-MS-encapsulated (MS- $\beta$ Gal) or soluble (sol. $\beta$-Gal) $\beta$-gal protein for $4 \mathrm{~h}$ at $37^{\circ} \mathrm{C}$ followed by maturation with LPS for $24 \mathrm{~h}$. BM-DC loaded with empty PLGA-MS (MS-E) or without Ag loading (w/o Ag) were used as controls. After washing, $3 \times 10^{5} \mathrm{BM}$-DC were injected intravenously into C57BL/6 mice (three mice per group). After 6, 10, and 16 days of immunization, PBL from each mouse were collected to detect ex vivo I8V-specific CD8 ${ }^{+}$ $\mathrm{T}$ cells in peripheral blood by I8V-tetramer staining. The flow cytometric analysis of one representative mouse in each group on day 6 are shown in (A). The numbers in the dot plots represent the mean percentage of I8V-tetramer positive $\mathrm{CD} 8^{+} \mathrm{T}$ cells of each group (three mice) \pm S.E.M. The kinetic analysis of the CTL responses is shown in (B). On day 16 after immunization, splenocytes from each mouse were restimulated with I8V peptide for 6 days in vitro and then analyzed by I8V-tetramer staining (C) and ${ }^{51} \mathrm{Cr}$ release assay using EL-4 cells pulsed with (closed symbols) or without I8V peptide (open symbols) as targets (D). Each symbol represents one mouse; results are averaged from two independent experiments. 
we stained BM-DC for CD54, CD86 and H-2IA ${ }^{\mathrm{b}}$ before and after uptake of PLGA-MS. However, no significant change was revealed by flow cytometric analysis (Fig. 4).

To monitor antigen-specific CTL generation in mice, we used adoptive transfer of bone marrow-derived DC in mice. For this purpose, we prepared PLGA-MS that contained the model antigen $\beta$-galactosidase (MS- $\beta$ gal) or the $\mathrm{H}-2 \mathrm{~K}^{\mathrm{b}}$ restricted I8V epitope of $\beta$-gal (MS-I8V, residues 497-504). Then, CD11 ${ }^{+}$BM-DC were loaded with soluble I8V peptide, MS-I8V, soluble $\beta$-gal, MS- $\beta$ gal, or empty PLGA-MS (MS-E) and subsequently injected i.v. into C57/BL6 mice. The expansion of I8V-specific CTL in vivo was assessed on day 6 after inoculation by staining of PBL with $\mathrm{I} 8 \mathrm{~V} / \mathrm{H}-2 \mathrm{~K}^{\mathrm{b}}$ tetramers and anti-CD8 Ab. Flow cytometric analysis indicated that BM-DC loaded with MS-I8V were slightly more potent than BM-DC pulsed with the soluble I8V peptide (Fig. 5A). Interestingly, BM-DC loaded with MS- $\beta$ gal were much more effective than BM-DC charged with the soluble $\beta$-gal protein. A kinetic analysis of the CTL response over 16 days post inoculation revealed that the I8V-specific response was longer lasting when the peptide was delivered to BM-DC via PLGA-MS (Fig. 5B). On days 10 and 16 after inoculation, respectively, three and two times more I8V specific CTLs were detected when antigen delivery occurred with PLGA-MS. In addition to CTL specificity we also assessed cytolytic function. Splenocytes that were recovered on day 16 after vaccination were restimulated in vitro for 6 days with BM-DC loaded with I8V peptide. Staining with $\mathrm{I} 8 \mathrm{~V} / \mathrm{H}-2 \mathrm{~K}^{\mathrm{b}}$ tetramers revealed that the in vitro expansion did not distort the relative frequencies observed ex vivo (Fig. 5C). The cytolytic assays that were performed subsequently indicated that cytolytic activity correlated well with the frequency of I8V specific CTL, thus indicating that the generated CTL were functionally intact (Fig. 5D).

\section{Discussion}

The delivery of antigens to DC in a manner that allows the generation of strong and enduring antigen-specific CTL and $\mathrm{T}$ helper cell responses has been a persisting challenge for DC-based immunotherapy. While pulsing with peptides is convenient, it has the disadvantage that peptides remain bound to cell surface expressed class I molecules for only a few hours. Moreover, peptide-based immunotherapy will remain confined to a subgroup of patients that express the appropriate restriction elements. Another problem is the lack of specific $\mathrm{T}$ cell help because it is still quite difficult to identify class II epitopes; thus, the probability that appropriate classes I and II epitopes are available for a certain patient is very low. Therefore, most DC-based trials resorted to loading DC with a tumor-specific CTL epitope combined with a tumor-unrelated protein as a source of Th epitopes. This approach, however, is not optimal since specific $\mathrm{T}$ cell help is needed for the optimal induction of CTL [24].
A much better solution would be to load MoDC with protein antigens which have a high probability of containing both MHC classes I and II epitopes for a patient's ensemble of restriction elements. Unfortunately, it has been shown for murine and human DC that the cross-presentation of soluble proteins is extremely inefficient and we have confirmed this for the influenza virus matrix protein and human MoDC in vitro (Fig. 3) and for $\beta$-galactosidase and murine BM-DC in vivo (Fig. 5). Alternative methods for introducing proteins into the classes I and II pathways via transfection of mRNA or infection with viral vectors have been pursued, but problems with the preservation of DC integrity and function, with the transfection efficiency, the generation of anti-viral antibodies, and safety concerns have not yet been resolved.

In this study, we have investigated the loading of human MoDC with peptides and proteins via encapsulation into PLGA microparticles. This approach circumvents many of the aforementioned pitfalls. Safety issues should not arise with this approach as no nucleic acids or infectious processes are involved and since the PLGA polymer is clinically approved and biodegrades into acids that are rapidly metabolized. Previously, we have shown that crucial functions of MoDC were not negatively affected by the uptake of PLGAMS [20]. Here, we have investigated the question of specific classes I and II-restricted antigen presentation after loading of MoDC with PLGA-MS-encapsulated proteins and peptides. In agreement with previous studies with murine and human macrophages as well as murine dendritic cells, we also find for human MoDC that PLGA-MS-encapsulated proteins are very efficiently presented on class I (Fig. 3) and class II (Fig. 2) molecules. For both model proteins, tetanus toxoid and GST-Flu matrix, which were used for the investigation of class II and class I presentation, respectively, we compared the efficiency of presentation achieved with the soluble and PLGA-MS-encapsulated form. For class II presentation the enhancement of presentation afforded by delivery via PLGA-MS was only apparent when limiting amounts of tetanus toxoid (TT) were used and when MoDC were matured after antigen delivery. Nevertheless, a roughly 10 -fold increase in sensitivity was observed with two different detection systems (Fig. 2). A more striking advantage of PLGA-MS-mediated antigen delivery, however, is the efficient class I-restricted cross-presentation by MoDC. Crosspresentation of PLGA-MS-encapsulated GST-Flu matrix was at least 50-fold more efficient than that of soluble GST-Flu matrix protein (Fig. 3B). This impressive enhancement in cross-presentation is probably not a unique property of the PLGA polymer, but rather a general property of microparticles with a diameter of $0.1-5 \mu \mathrm{m}$ as similar findings have been made in murine dendritic cells and macrophages with iron oxide beads [25], latex beads [5], or polysaccharides [26] of a similar size.

In addition to facilitating and enhancing classes I and II-restricted presentation, loading MoDC with PLGA-MS affords a striking prolongation of antigen presentation. This property was apparent for class II-restricted presentation of 
tetanus toxoid when it was applied to MoDC at a low concentration (Fig. 2H), but it was even more prominent for class I-restricted cross-presentation of the M1 epitope of influenza virus matrix protein (Fig. 3A). Compared to loading with soluble M1 peptide, the loading with PLGA-MS achieved a duplication of the time period of M1 presentation (Fig. 3A). The efficient M1 presentation is interesting in the light of reports that cell-associated peptides cannot be crosspresented [27-29]. It appears that the encapsulation into PLGA-MS protects peptides from degradation which may be due to a release of PLGA-MS fiom the endo/lysosome into the cytosol, as was recently demonstrated for PLGA nanoparticles [30]. Presentation of the M1 epitope was detected up to three days after charging MoDC with PLGA-MSencapsultated GST-FluM protein. This is only half of the time period of M1 presentation which we achieved with PLGAMS encapsulated M1 peptide. However, keeping in mind that 500-3000 proteins may need to be degraded in order to generate one CTL epitope [31], the lower level and duration of presentation is not unexpected.

To investigate if this improvement of antigen presentation translates into better CTL responses in vivo, we examined the generation of CTL specific for the I8V peptide of $\beta$-galactosidase in the mouse model. Interestingly, the efficiency of CTL generation in vivo paralleled the characteristics of antigen presentation by MoDC in vitro (Fig. 5) in that PLGA-MS-mediated peptide delivery to BM-DC led to a significant increase in the number of I8V-specific CTL on day 6 post vaccination, which was augmented to a three-fold increase on day 10 after vaccination (Fig. 5B). In accordance with our in vitro results with human MoDC, no significant CTL response could be obtained when BM-DC were loaded with soluble $\beta$-galactosidase protein, but almost $5 \%$ of $\mathrm{CD}^{+}$ $\mathrm{T}$ cells were specific for the I8V peptide on day 6 after vaccination with BM-DC when they were loaded with PLGAMS encapsulated P-galactosidase. This protocol of DC-based vaccination afforded not only a strong increase in CTL numbers, but the CTL were also functional as determined by cytolytic tests (Fig. 5D).

Taken together, we show in this study that antigen delivery to human MoDC by PLGA-MS has important advantages for DC-based vaccination in vitro and in vivo. Since the protocols for MoDC generation used in this study are suitable for clinical application and since PLGA is a pharmaceutically accepted material, our investigations set the stage for testing this technique of antigen delivery in clinical trials of DCbased immunotherapy against tumors and infectious diseases.

\section{Acknowledgements}

We thank Burkhard Ludewig for experimental advice, Stephan Ludwig for kindly providing a GST-matrix expression construct, and Phillipe Krebs for the generous gift of MHC tetramers. Dr. Markus Fopp and the personnel of the St. Gallen Blood Bank are acknowledged for providing blood products. This work was supported by grants from the Cancer League St. Gallen-Appenzell, Swiss Cancer League (01099-02-2001), Foundation Propter Homines Vaduz Liechtenstein, Cancer Research Institute, and Deutsche Krebshilfe (10-2156).

\section{References}

[1] Banchereau J, Briere F, Caux C, Davoust J, Lebecque S, Liu YT, et al. Immunobiology of dendritic cells. Annu Rev Immunol 2000;18:767-811.

[2] Jung S, Unutmaz D, Wong P, Sano GI, DelosSantos K, Sparwasser $\mathrm{T}$, et al. In vivo depletion of $\mathrm{CD} 11 \mathrm{c}(+)$ dendritic cells abrogates priming of CD8(+) T cells by exogenous cell-associated antigens. Immunity 2002;17(2):211-20.

[3] Lanzavecchia A. Mechanisms of antigen uptake for presentation. Curr Opin Immunol 1996;8(3):348-54.

[4] Heath WR, Belz GT, Behrens G.M.N., Smith CM, Forehan SP, Parish IA, et al. Cross-presentation, dendritic cell subsets, and the generation of immunity to cellular antigens. Immunol Rev 2004;199:9-26.

[5] Shen Z, Reznikoff G, Dranoff G, Rock KL. Cloned dendritic cells can present exogenous antigens on both MHC class I and class II molecules. J Immunol 1997;158(6):2723-30.

[6] Schuler G, SchulerThurner B, Steinman RM. The use of dendritic cells in cancer immunotherapy. Curr Opin Immunol 2003;15(2):138-47.

[7] Sallusto F, Lanzavecchia A. Efficient presentation of soluble antigen by cultured human dendritic cells is maintained by granulocyte/macrophage colony-stimulating factor plus interleukin 4 and downregulated by tumor necrosis factor alpha. J Exp Med 1994;179(4):1109-18.

[8] Thurner B, Roder C, Dieckmann D, Heuer M, Kruse M, Glaser A, et al. Generation of large numbers of fully mature and stable dendritic cells from leukapheresis products for clinical application. J Immunol Methods 1999;223(1):1-15.

[9] Ludewig B, McCoy K, Pericin M, Ochsenbein AF, Dumrese T, Odermatt $\mathrm{B}$, et al. Rapid peptide turnover and inefficient presentation of exogenous antigen critically limit the activation of self-reactive CTL by dendritic cells. J Immunol 2001;166(6):3678-87.

[10] Johansen P, Men Y, Merkle HP, Gander B. Revisiting PLA/PLGA microspheres: an analysis of their potential in parenteral vaccination. Eur J Pharm Biopharm 2000;50(1):129-46.

[11] Men Y, Thomasin C, Merkle H, Gander B, Corradin G. A single administration of tetanus toxoid in biodegradable microspheres elicits $\mathrm{T}$ cell and antibody responses similar or superior to those obtained with aluminum hydroxide. Vaccine 1995;13(7):683-9.

[12] Men Y, Gander B, Merkle HP, Corradin G. Induction of sustained and elevated immune responses to weakly immunogenic synthetic malarial peptides by encapsulation in biodegradable polymer microspheres. Vaccine 1996;14(15):1442-50.

[13] Shi L, Caulfield MJ, Chern RT, Wilson RA, Sanyal G, Volkin DB. Pharmaceutical and immunological evaluation of a single-shot hepatitis B vaccine formulated with PLGA microspheres. J Pharm Sci 2002;91(4):1019-35.

[14] Men Y, Tamber H, Audran R, Gander B, Corradin G. Induction of a cy totoxic $\mathrm{T}$ lymphocyte response by immunization with a malaria specific CTL peptide entrapped in biodegradable polymer microspheres. Vaccine 1997;15(12-13):1405-12.

[15] Partidos CD, Vohra P, Jones D, Farrar G, Steward MW. CTL responses induced by a single immunization with peptide encapsulated in biodegradable microparticles. J Immunol Methods 1997;206(1-2):143-51.

[16] Otten G, Schaefer M, Greer C, Calderon-Cacia M, Coit D, Kazzaz $\mathrm{J}$, et al. Induction of broad and potent anti-human immunodeficiency 
virus immune responses in rhesus macaques by priming with a DNA vaccine and boosting with protein-adsorbed polylactide coglycolide microparticles. J Virol 2003;77(10):6087-92.

[17] Cabarrocas J, Bauer J, Piaggio E, Liblau R, Lassmann H. Effective and selective immune surveillance of the brain by MHC class I-restricted cytotoxic T lymphocytes. Eur J Immunol 2003;33(5):1174-82.

[18] Thomasin C, Corradin G, Men Y, Merkle HP, Gander B. Tetanus toxoid and synthetic malaria antigen containing poly(lactide)/ poly(lactide-co-glycolide) microspheres: importance of polymer degradation and antigen release for immune response. J Control Release 1996;41:131-45.

[19] Peyre M, Fleck R, Hockley D, Gander B, Sesardic D. In vivo uptake of an experimental microencapsulated diphtheria vaccine following sub-cutaneous immunisation. Vaccine 2004;22(19):2430-7.

[20] Waeckerle-Men Y, Scandella E, Allmen EU, Ludewig B, Gillessen S, Merkle HP, et al. Phenotype and functional analysis of human monocyte-derived dendritic cells loaded with biodegradable poly(lactide-co-glycolide) microspheres for immunotherapy. J Immunol Method 2004;287(1-2):109-24.

[21] Men Y, Audran R, Thomasin C, Eberl G, Demotz S, Merkle HP, et al. MHC class I- and class H-restricted processing and presentation of microencapsulated antigens. Vaccine 1999;17(9-10):1047-56.

[22] Audran R, Peter K, Dannull J, Men Y, Groettrup M, Gander B, et al. Micro-encapsulation of peptides prolongs their presentation to cytotoxic $\mathrm{T}$ cells by antigen presenting cells in vitro. Vaccine 2003;21(11-12):1250-5.

[23] Scandella E, Men Y, Gillessen S, Forster R, Groettrup M. Prostaglandin E2 is a key factor for CCR7 surface expression and migration of monocyte-de rived dendritic cells. Blood 2002;100(4): 1354-61.
[24] Ossendorp F, Mengede E, Camps M, Filius R, Melief CJM. Specific $\mathrm{T}$ helper cell requirement for optimal induction of cytotoxic $\mathrm{T}$ lymphocytes against major histocompatibility complex class $\mathrm{H}$ negative tumors. J Exp Med 1998;187(5):693-702.

[25] Kovacsovics-Bankowski M, Clark K, Benacerraf B, Rock KL. Efficient major histocompatibility complex class I presentation of exogenous antigen upon phagocytosis by macrophages. Proc Natl Acad Sci USA 1993;90(11):4942-6.

[26] Ikuta Y, Katayama N, Wang LJ, Okugawa T, Takahashi Y, Schmitt $\mathrm{M}$, et al. Presentation of a major histocompatibility complex class 1-binding peptide by monocyte-derived dendritic cells incorporating hydrophobized polysaccharide-truncated HER2 protein complex: implications for a polyvalent immuno-cell therapy. Blood 2002;99(10):3717-24.

[27] Norbury CC, Basta S, Donohue KB, Tscharke DC, Princiotta MF, Berglund $\mathrm{P}$, et al. CD8(+)T cell cross-priming via transfer of proteasome substrates. Science 2004;304:1318-21.

[28] Wolkers MC, Brouwenstijn N, Bakker AH, Toebes M, Schumacher TN. Antigen bias in $\mathrm{T}$ cell cross-priming. Science 2004;304(5675):1314-7.

[29] Shen LJ, Rock KL. Cellular protein is the source of crosspriming antigen in vivo. Proc Nat Acad Sci USA 2004;101(9):303540.

[30] Panyam J, Zhou WZ, Prabha S, Sahoo SK, Labhasetwar V. Rapid endo-lysosomal escape of poly(DL-lactide-co-glycolide) nanoparticles: implications for drug and gene delivery. FASEB J 2002;16(10):1217-26.

[31] Princiotta MF, Finzi D, Qian SB, Gibbs J, Schuchmann S, Buttgereit F, et al. Quantitating protein synthesis, degradation, and endogenous antigen processing. Immunity 2003;18(3):343-54. 